\title{
Different roles of Staphylococcus aureus enterotoxin in different subtypes of nasal polyps
}

\author{
KE-JIA CHENG, SHEN-QING WANG and YING-YING XU \\ Department of Otolaryngology, The First Affiliated Hospital, College of Medicine, \\ Zhejiang University, Hangzhou, Zhejiang 310003, P.R. China
}

Received July 28, 2015; Accepted September 13, 2016

DOI: $10.3892 /$ etm.2016.3951

\begin{abstract}
Chronic rhinosinusitis with nasal polyps (CRSwNP) is a multifactorial disease. The pathogenesis of CRSwNP remains unclear. This study was designed to investigate the role of inflammation and Staphylococcus aureus enterotoxin (SE) in this disease. The study included a total of 74 patients with CRSwNP and 6 controls. A serum Phadiatop assay was conducted to detect atopy status, and serum eosinophil cationic protein (ECP) and total immunoglobulin (Ig)E levels were determined using ELISA. SEA, SEB, total IgE, ECP and myeloperoxidase (MPO) levels in nasal tissue supernatant were measured using ELISA. The results indicated that $15(22.1 \%)$ patients had systemic allergies. On the basis of the ECP/MPO ratio, the patients were divided into an eosinophilic CRSwNP group $(n=18)$ and a non-eosinophilic CRSwNP group $(n=56)$. The total ECP/MPO ratio was 0.572 , with a notable bias toward neutrophilic inflammation. The supernatant ECP and MPO levels were elevated in the CRSwNP group compared with the control group, but no significant difference in the serum total IgE and ECP levels were observed between the CRSwNP and control groups. In addition, the non-eosinophilic and eosinophilic CRSwNP groups showed significant elevations in supernatant total $\operatorname{IgE}$, SEA and SEB levels compared with the control group. Thus, it may be concluded that allergy is a common pathogenesis of CRSwNP, and neutrophilic inflammation is present in most Chinese CRSwNP patients. Additionally, local indicators reflect the inflammatory status more accurately than do serum indicators. SEs may act as an infection factor rather than as a superantigen in Chinese non-eosinophilic CRSwNP
\end{abstract}

Correspondence to: $\mathrm{Dr}$ Ying-Ying $\mathrm{Xu}$, Department of Otolaryngology, The First Affiliated Hospital, College of Medicine, Zhejiang University, 79 Qingchun Road, Hangzhou, Zhejiang 310003, P.R. China

E-mail: xuyingying128@163.com

Key words: chronic rhinosinusitis with nasal polyps, superantigen, Staphylococcus aureus enterotoxin, eosinophil cationic protein, myeloperoxidase patients. Thus, long-term antibiotic therapy may be an option for Chinese non-eosinophilic CRSwNP patients.

\section{Introduction}

Chronic rhinosinusitis (CRS) is a common disease of the nasal cavity and sinuses that affects millions of people worldwide (1). CRS can be divided into two subtypes: CRS with nasal polyps (CRSwNP) and CRS without nasal polyps (CRSsNP) (2). Compared with CRSsNP, CRSwNP has a more complex pathogenesis and usually requires surgery (3). At present, the exact origin of CRSwNP remains unclear. Inappropriate therapeutic options lead to a high recurrence rate (4).

According to the type of inflammatory cell infiltration, CRSwNP can be divided into two subgroups: Eosinophilic and non-eosinophilic or neutrophilic (5). Non-eosinophilic CRSwNP always presents with neutrophil-predominant inflammation (6). The subgroups have a variety of pathogeneses and may require different therapeutic options. Eosinophilic subgroup is considered to be induced by Th2 cells, with interleukin (IL)-5 as major cytokine, resulting in increased eosinophil survival and an eosinophilic type of inflammation. The predominant T-effector cell in non-eosinophilic subgroup is the Th17 cell, and resulting in a predominance of neutrophils $(7,8)$. In a previous study, the authors suggested that Caucasian patients with CRSwNP usually manifest with eosinophilic CRSwNP, whereas Chinese patients always show non-eosinophilic inflammation (9). However, this view remains controversial. In the present study, the levels of eosinophil cationic protein (ECP), which is released by active eosinophils, and myeloperoxidase (MPO) were tested to detect the subtype of CRSwNP in Chinese patients.

Staphylococcus aureus and S. aureus enterotoxins (SEs) are common pathogens of CRS. However, the exact underlying pathogenesis remains unclear. SEs are composed mainly of SEA, SEB, SEC, SED and shock syndrome toxin (TSST-1) (10). In Caucasian patients with CRSwNP, SEs have been suggested to act as classic allergens or superantigens, which are able to activate millions of T and B lymphocytes (11). Superantigens elicit the production of high levels of allergen-specific immunoglobulin (Ig)E, and are usually associated with the elevation of total $\mathrm{IgE}$ and eosinophilic inflammation (12). However, evidence is lacking concerning the role of superantigens for SEs in Chinese patients with CRSwNP. In non-eosinophilic 
CRSwNP, SEs may act as an infection factor; this hypothesis was tested in the current study.

\section{Materials and methods}

Subjects. A total of 74 patients with CRSwNP who had undergone functional endoscopic sinus surgery at the First Affiliated Hospital of the College of Medicine, Zhejiang University (Hangzhou, China) from July to December 2013 (50 males and 24 females; mean age, 42.35 years) were included in the present study. The diagnosis of CRSwNP was based on European Paediatric Ophthalmological Society guidelines (13) and was confirmed by pathological diagnosis after surgery. Patients who used corticosteroids or antihistamines for the previous 4 weeks were excluded from this study, as were patients with asthma. The duration of CRSwNP in the patients ranged from 3 months to 20 years. Six patients who underwent septoplasty or sinus cystectomy were designated as the control group.

The present research was approved by the Ethics Committee of the First Affiliated Hospital of Zhejiang University. Each patient supplied written informed consent.

Atopy status detection. Atopy status was measured in vitro by testing the serum using the Phadiatop test and UniCAP100 automated system (Phadia AB; Thermo Fisher Scientific, Inc., Waltham, MA, USA) according to the manufacturer's instructions. The test included the most common aeroallergens. The cut-off value was set at $0.35 \mathrm{KU} / 1$.

Homogenate supernatant of the nasal tissues. All of the nasal tissues (polyps or turbinates) were collected at the time of surgery, were placed immediately in liquid nitrogen, and then stored at $-80^{\circ} \mathrm{C}$. Specimens were first thawed in saline $(0.1 \mathrm{~g}$ in $1 \mathrm{ml}$ ) and processed in a tissue homogenizer. The suspensions were then centrifuged for $5 \mathrm{~min}$ at $1,006.2 \times \mathrm{g}$. The supernatants were collected and stored at $-80^{\circ} \mathrm{C}$ prior to analysis.

Total IgE and ECP levels in the serum and supernatant. Total IgE levels in the serum and nasal tissue supernatant were detected by enzyme-linked immunosorbent assay (\#BMS209; ELISA; eBioscience, Vienna, Austria). First, the number of microwell strips required to test the desired number of samples plus the appropriate number of wells needed to run the blanks and standards were determined. Each sample, standard, blank and optional control sample was assayed in duplicate. Next, horseradish peroxidase (HRP)-conjugated antibody was prepared. The microwell strips were washed twice with $\sim 400 \mu \mathrm{l}$ Wash Buffer per well with thorough aspiration of the microwell contents between washes. Then, $100 \mu 1$ Assay Buffer (1X) was added in duplicate to the blank wells, followed by the addition of $90 \mu \mathrm{l}$ Assay Buffer (1X) and $10 \mu \mathrm{l}$ each sample in duplicate to the sample wells. To each well, including the blank wells, $50 \mu$ l diluted HRP-conjugated antibody was then added. The wells were covered with an adhesive film and incubated at room temperature $\left(18-25^{\circ} \mathrm{C}\right)$ for $1 \mathrm{~h}$ on a microplate shaker set at $400 \mathrm{rpm}$. Next, the adhesive film and the liquid in the wells were removed. The microwell strips were washed four times with wash buffer according to the test protocol. Next, $100 \mu \mathrm{l}$ Tetramethylbenzidine Substrate Solution was pipetted into each well. The microwell strips were then incubated at room temperature $\left(18-25^{\circ} \mathrm{C}\right)$ for $30 \mathrm{~min}$, taking care to avoid direct exposure to intense light. The enzyme reaction was stopped by quickly pipetting $100 \mu \mathrm{l}$ Stop Solution into each well. The absorbance of each microwell was read on a spectrophotometer using $450 \mathrm{~nm}$ as the primary wavelength, and the results were calculated.

ECP levels were also measured by ELISA (\#SK00128-01; Aviscera Bioscience, Inc., Santa Clara, CA, USA), according to the manufacturer protocol.

SEA, SEB and MPO levels in the supernatant. SEA [\#BIO(TW)-E01(Hu)-00254] and SEB [\#BIO(TW)E01(Hu)-00253] levels were measured using ELISA (R\&D Systems, Inc., Minneapolis, MN, USA). MPO levels were also detected using an ELISA kit (\#BMS2038INST; eBioscience) according to the manufacturer's protocol.

Statistical analysis. All data analyses were performed using SPSS for Windows 20.0 (IBM SPSS, Armonk, NY, USA). Considering the non-normal distributions of the parameters, median (25-75\% percentile) was used to describe the data. Differences in each group were compared using the Mann-Whitney $\mathrm{U}$ test. $\mathrm{P}<0.05$ was considered to indicate a statistically significant difference.

\section{Results}

Atopy status of CRSwNP patients. Serum testing was conducted for $68 \mathrm{CRSwNP}$ patients and 6 controls. Among the CRSwNP patients 15 (22.1\%) showed positive results in the Phadiatop test, and none of the controls exhibited atopy.

ECP and MPO levels in nasal tissue supernatant. All of the CRSwNP patients and controls underwent the supernatant tests. As described previously, eosinophilic CRSwNP is characterized by a supernatant ECP/MPO ratio >2 (14). According to this criterion, 18 patients were assigned to an eosinophilic CRSwNP group, and the others were assigned to the non-eosinophilic CRSwNP group. The total ECP/MPO ratio was 0.572 , with a notable bias toward neutrophilic inflammation. Table I lists the ECP and MPO levels in the supernatants of the three groups. The supernatant ECP level was significantly elevated in the non-eosinophilic and eosinophilic CRSwNP groups compared with that in the control group $(\mathrm{P}<0.022$ and $\mathrm{P}<0.001$, respectively). The supernatant MPO level was significantly elevated in the non-eosinophilic CRSwNP group compared with the control group $(\mathrm{P}=0.001)$ (Fig. 1).

Total IgE and ECP levels in the serum. In the CRSwNP group, the ECP level in the serum was $10.55 \mu \mathrm{g} / 1$ (range, 6.92-22.60 $\mu \mathrm{g} / \mathrm{l})$. Compared with the control group, the CRSwNP group, including eosinophilic and non-eosinophilic subgroups, showed no significant elevation in serum ECP level. In the CRSwNP group, the total IgE level in the serum was $45.70 \mathrm{KU} / 1(20.00-152.75 \mathrm{KU} / \mathrm{l})$. Compared with the control group, the CRSwNP group showed no significant difference in the serum total IgE level. Data are listed in Table II and Fig. 2.

Total IgE and SE levels in the supernatant. Table III summarizes total IgE and SE levels in the supernatant. Compared 
Table I. ECP and MPO levels in the nasal tissue supernatant.

\begin{tabular}{|c|c|c|c|c|}
\hline Group & $\mathrm{ECP}(\mu \mathrm{g} / \mathrm{l})$ & P-value & MPO $(\mu \mathrm{g} / 1)$ & P-value \\
\hline Non-eosinophilic CRSwNP & $36.31(20.70-69.15)$ & 0.022 & 72.64 (34.65-178.37) & 0.001 \\
\hline Eosinophilic CRSwNP & $121.38(63.59-164.57)$ & $<0.001$ & $26.09(18.14-49.57)$ & 0.820 \\
\hline Control & $15.00(13.21-31.32)$ & & $27.34(14.92-30.26)$ & \\
\hline
\end{tabular}

ECP, eosinophil cationic protein; MPO, myeloperoxidase; CRSwNP, chronic rhinosinusitis with nasal polyps.

Table II. Serum total IgE and ECP level in different groups.

\begin{tabular}{lccc}
\hline Group & Serum total IgE $(\mathrm{KU} / \mathrm{l})$ & P-value & Serum ECP $(\mu \mathrm{g} / \mathrm{l})$ \\
\hline CRSwNP & $45.70(20.00-152.75)$ & 0.945 & $10.55(6.92-22.60)$ \\
Non-eosinophilic CRSwNP & $42.65(19.88-140.75)$ & 0.990 & $9.49(6.18-17.28)$ \\
Eosinophilic CRSwNP & $79.65(24.90-194.00)$ & 0.820 & $14.45(9.80-42.80)$ \\
Control & $46.80(17.08-296.75)$ & & $13.85(9.56-26.58)$ \\
\hline
\end{tabular}

IgE, immunoglobulin E; ECP, eosinophil cationic protein; CRSwNP, chronic rhinosinusitis with nasal polyps.

A

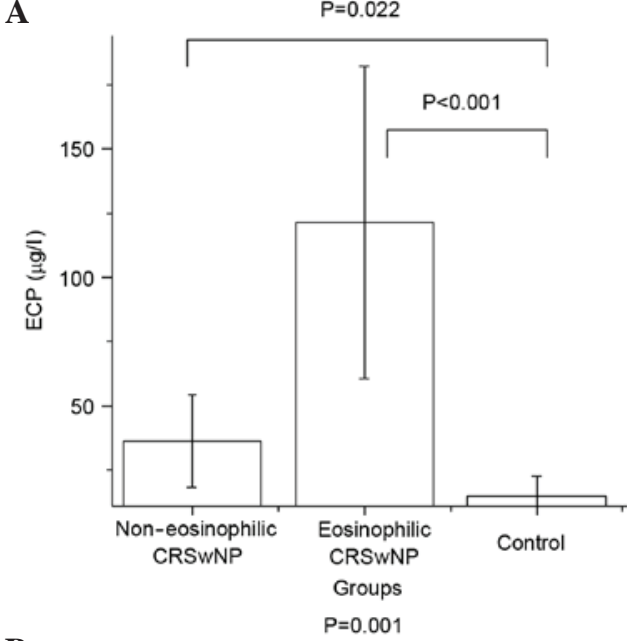

B

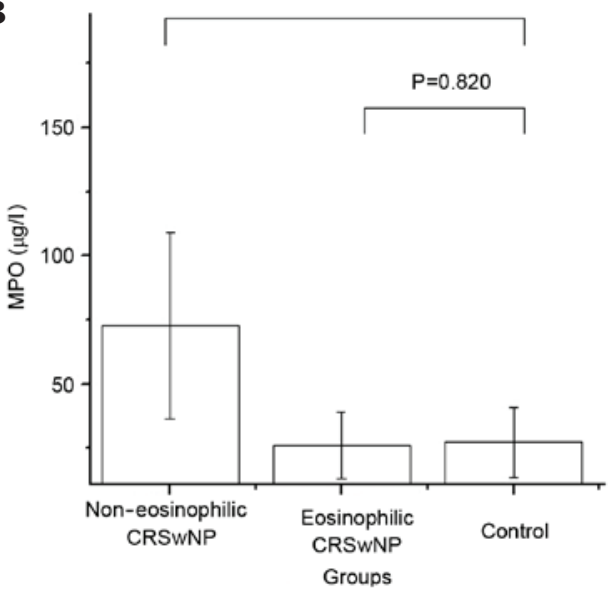

Figure 1. ECP and MPO levels in nasal tissue supernatants. (A) The supernatant ECP level was significantly elevated in the non-eosinophilic and eosinophilic CRSwNP groups compared with that in the control group. (B) The supernatant MPO level was significantly elevated in the non-eosinophilic CRSwNP group and was also elevated in the eosinophilic CRSwNP group, albeit not significantly so. ECP, eosinophil cationic protein; MPO, myeloperoxidase; CRSwNP, chronic rhinosinusitis with nasal polyps. with the control group, the non-eosinophilic and eosinophilic CRSwNP groups showed significant elevations in supernatant total IgE levels $(\mathrm{P}=0.013$ and $\mathrm{P}<0.001$, respectively). The eosinophilic CRSwNP group also showed a significant elevation in supernatant total IgE level compared with that in the non-eosinophilic group $(\mathrm{P}<0.001)$. Compared with the control group, the non-eosinophilic and eosinophilic CRSwNP groups showed significant elevations in the supernatant SEA levels $(\mathrm{P}=0.006$ and $\mathrm{P}=0.04$, respectively) and SEB levels $(\mathrm{P}=0.001$ and $\mathrm{P}=0.004$, respectively). The differences in supernatant SEA and SEB levels between the non-eosinophilic and eosinophilic CRSwNP groups were not found to be statistically significant (Fig. 3).

\section{Discussion}

CRSwNP is a complex disease that presents challenges to rhinologists. Its pathogenesis remains unclear, with allergy, infection, inflammation, anatomic abnormality, bacterial superantigen and biofilm being suggested (15). The recurrence rate for CRSwNP is high after surgery or drug treatment in certain patients (16). In the past, allergy was considered to be an important pathogeny of CRSwNP (17). Allergic CRSwNP showed a worse therapeutic response than non-allergic CRSwNP in patients treated with budesonide nasal spray (18). However, its underlying pathogenesis remains unclear (19). In the present study, 15 (22.1\%) CRSwNP patients showed atopy, representing a higher incidence than that for healthy people. This finding suggests that atopy remains a common pathogenesis of CRSwNP, a viewpoint that is shared with two previous studies $(20,21)$.

Previously, CRSwNP was regarded as being associated with T helper (Th)2-predominant inflammation, with increased eosinophil infiltration and IgE elevation (22). In the present study, the serum total IgE and ECP levels were not observed to be increased in the CRSwNP groups compared with those 
Table III. Total IgE, SEA and SEB level in the supernatant.

\begin{tabular}{|c|c|c|c|c|c|c|}
\hline Group & Total IgE (KU/l) & P-value & $\mathrm{SEA}(\mu \mathrm{g} / 1)$ & P-value & $\mathrm{SEB}(\mu \mathrm{g} / \mathrm{l})$ & P-value \\
\hline Non-eosinophilic CRSwNP & $22.72(9.22-36.22)$ & $0.013^{\mathrm{a}}$ & $77.99(43.00-132.45)$ & $0.006^{\mathrm{a}}$ & $9.85(4.73-17.04)$ & $0.001^{\mathrm{a}}$ \\
\hline Eosinophilic CRSwNP & $50.15(34.28-90.74)$ & $<0.001^{\mathrm{b}}$ & $71.56(29.82-109.21)$ & $0.541^{\mathrm{b}}$ & $10.97(5.00-27.99)$ & $0.597^{\mathrm{b}}$ \\
\hline Control & $7.71(5.25-8.79)$ & $<0.001^{\mathrm{c}}$ & $27.38(12.58-53.41)$ & $0.04^{\mathrm{c}}$ & $3.28(1.23-5.13)$ & $0.004^{\mathrm{c}}$ \\
\hline
\end{tabular}

${ }^{a}$ Non-eosinophilic CRSwNP group vs. control group, ${ }^{b}$ non-eosinophilic CRSwNP group vs. eosinophilic CRSwNP group, ${ }^{c}$ eosinophilic CRSwNP group vs. control group. IgE, immunoglobulin E; SEA, Staphylococcus aureus enterotoxin A; SEB, S. aureus enterotoxin B; CRSwNP, chronic rhinosinusitis with nasal polyps.

A

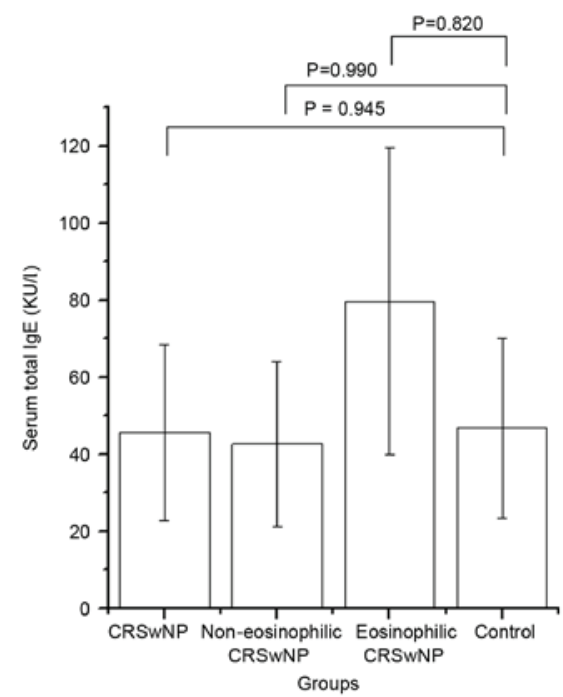

B

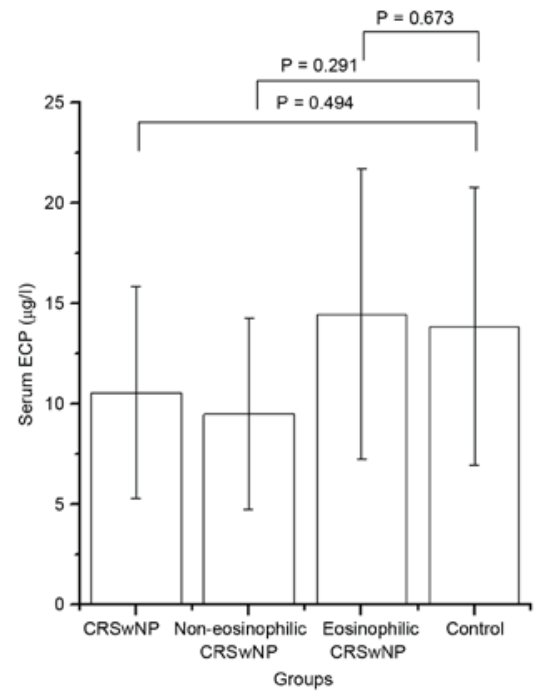

Figure 2. Total IgE and ECP levels in the serum. (A) The CRSwNP group showed no significant difference in serum total IgE level compared with the control group. (B) The CRSwNP group, including the eosinophilic and non-eosinophilic groups, showed no significant elevation in serum ECP level. CRSwNP, chronic rhinosinusitis with nasal polyps; IgE, immunoglobulin E; $\mathrm{ECP}$, eosinophil cationic protein.

in the control group. It is thus suggested that these indicators in local tissues or nasal lavage might be more sensitive than those in the serum.

Currently, CRSwNP is subdivided into two subgroups: Eosinophilic and non-eosinophilic. Different CRS endotypes
$\mathbf{A}$

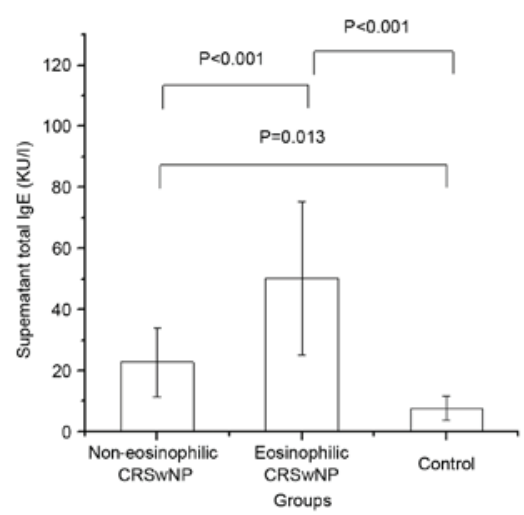

B

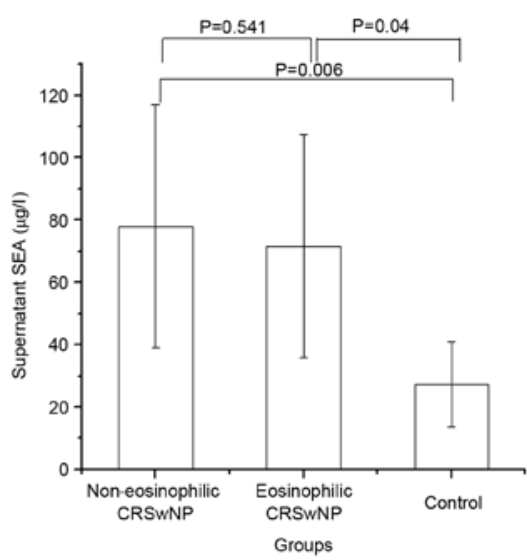

C

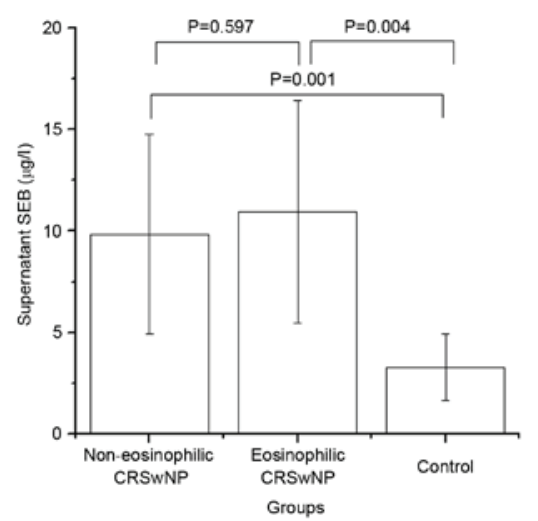

Figure 3. Total IgE and SE levels in nasal tissue supernatant. (A) Compared with the control, the non-eosinophilic and eosinophilic CRSwNP groups showed significant increases in supernatant total IgE levels. The eosinophilic CRSwNP group also showed a significant elevation in supernatant total $\mathrm{IgE}$ level compared with that in the non-eosinophilic group. Compared with the control, the non-eosinophilic and eosinophilic CRSwNP groups showed significant elevations in supernatant (B) SEA and (C) SEB levels. CRSwNP, chronic rhinosinusitis with nasal polyps; IgE, immunoglobulin E; SEA, Staphylococcus aureus enterotoxin A; SEB, S. aureus enterotoxin B. 
can be characterized by the differences in responsiveness to treatments (23). Thus, the exact diagnosis of endotypes is important. Eosinophilic CRSwNP is an inflammatory disease characterized by increased numbers of eosinophils, in particular, fibroblasts, mast cells, goblet cells and Th2 lymphocytes (24). Eosinophilic CRSwNP is usually associated with IgE IL-5 elevation, classic allergy and superantigens (25). Eosinophilic CRSwNP has been found in the majority of CRSwNP patients who are Caucasian (9). Non-eosinophilic CRSwNP exhibits neutrophilic inflammation in which neutrophil recruitment into the sinus effusion is mediated by the upregulation of adhesion molecules of the vascular endothelium induced by IL-17 and by the enhanced secretion of IL- 8 from epithelial cells and neutrophils $(26,27)$. Additionally, non-eosinophilic CRSwNP is usually characterized by Th1/Th17-shifted immunity (14). Non-eosinophilic inflammation is common in Chinese CRSwNP patients (28). The difference in endotypes is not associated with only racial differences; in a previous study, the cytokine profiles in Japanese patients with CRSwNP were found to be similar to those of European patients with CRS (29).

In the present study, 56 (75.7\%) of the CRSwNP patients were considered to have non-eosinophilic CRSwNP, and the others were considered to have eosinophilic CRSwNP. This suggests that non-eosinophilic inflammation plays an important role in CRSwNP in Chinese patients, which is in agreement with the findings of previous research $(30,31)$. In the current study, the supernatant ECP and MPO levels were elevated in the eosinophilic CRSwNP groups. It may be inferred that both eosinophils and neutrophils are involved in the pathogenesis of eosinophilic CRSwNP, with eosinophils being the main functional cells in the eosinophilic group.

Many studies have suggested a potential role for SEs in the etiology and pathogenesis of CRSwNP $(32,33)$. SEs usually act as superantigens or classic allergens, which are able to stimulate the elevation of local total IgE and allergen-specific IgE levels and eosinophilic inflammation (34). In the current study, the supernatant total IgE, SEA and SEB levels were upregulated in both the eosinophilic and non-eosinophilic CRSwNP groups. The supernatant total IgE level was significantly higher in the eosinophilic group compared with that in the non-eosinophilic group. These results implied that SEs are involved in the allergic pathogenesis of the eosinophilic CRSwNP endotype. However, in the non-eosinophilic endotype, SEs may play an alternative role in the pathogenesis of this disease. Consistent with the hygiene hypothesis, SEs may act as infection factors, but not allergens, due to the frequent exposure to bacteria and viruses.

In conclusion, allergy remains a common factor in the pathogenesis of CRSwNP. Neutrophilic inflammation is present in the majority of Chinese CRSwNP patients. Additionally, local indicators appear to reflect the inflammatory status more accurately than do serum indicators. SEs may act as an infection factor rather than as a superantigen in Chinese patients with non-eosinophilic CRSwNP. Thus, long-term antibiotic therapy may be an option for Chinese patients with non-eosinophilic CRSwNP.

\section{Acknowledgements}

The study was supported by Zhejiang Province Public Welfare Funds (grant no. 2014C33203).
The English in this document has been checked by at least two professional editors, both native speakers of English. For a certificate, please see: http://www.textcheck.com/certificate/LMh1Rb.

\section{References}

1. Bhattacharyya N and Lee LN: Evaluating the diagnosis of chronic rhinosinusitis based on clinical guidelines and endoscopy. Otolaryngol Head Neck Surg 143: 147-151, 2010.

2. Polzehl D, Moeller P, Riechelmann H and Perner S: Distinct features of chronic rhinosinusitis with and without nasal polyps. Allergy 61: 1275-1279, 2006.

3. Benninger MS, Ferguson BJ, Hadley JA, Hamilos DL, Jacobs M, Kennedy DW, Lanza DC, Marple BF, Osguthorpe JD, Stankiewicz JA, et al: Adult chronic rhinosinusitis: Definitions, diagnosis, epidemiology, and pathophysiology. Otolaryngol Head Neck Surg 129 (3 Suppl): S1-S32, 2003.

4. Tosun F, Arslan HH, Karslioglu Y, Deveci MS and Durmaz A: Relationship between postoperative recurrence rate and eosinophil density of nasal polyps. Ann Otol Rhinol Laryngol 119: 455-459, 2010.

5. Hirotsu M, Kikuchi K, Kusunoki T, Kase K, Ono N and Ikeda K: Comparison of bacterial examinations between eosinophilic and neutrophilic chronic rhinosinusitis with nasal polyps. Acta Otolaryngol 131: 997-1001, 2011.

6. Ba L, Zhang N, Meng J, Zhang J, Lin P, Zhou P, Liu S and Bachert C: The association between bacterial colonization and inflammatory pattern in Chinese chronic rhinosinusitis patients with nasal polyps. Allergy 66: 1296-303, 2011.

7. Fokkens W, Lund V and Mullol J; European Position Paper on Rhinosinusitis and Nasal Polyps Group: EP3OS 2007: European position paper on rhinosinusitis and nasal polyps 2007. A summary for otorhinolaryngologists. Rhinology 45: 97-101, 2007.

8. Meltzer EO, Hamilos DL, Hadley JA, Lanza DC, Marple BF, Nicklas RA, Adinoff AD, Bachert C, Borish L, Chinchilli M, et al; Rhinosinusitis Initiative: Rhinosinusitis: Developing guidance for clinical trials. J Allergy Clin Immunol 118 (5 Suppl): S17-S61, 2006.

9. Bachert C, Zhang N, van Zele T and Gevaert P: Chronic rhinosinusitis: From one disease to different phenotypes. Pediatr Allergy Immunol 23 (Suppl 22): S2-S4, 2012.

10. Patou J, Gevaert P, Van Zele T, Holtappels G, van Cauwenberge P and Bachert C: Staphylococcus aureus enterotoxin B, protein A, and lipoteichoic acid stimulations in nasal polyps. J Allergy Clin Immunol 121: 110-115, 2008.

11. Bernstein JM and Kansal R: Superantigen hypothesis for the early development of chronic hyperplastic sinusitis with massive nasal polyposis. Curr Opin Otolaryngol Head Neck Surg 13: 39-44, 2005.

12. Van Zele T, Gevaert P, Holtappels G, van Cauwenberge P and Bachert C: Local immunoglobulin production in nasal polyposis is modulated by superantigens. Clin Exp Allergy 37: 1840-1847, 2007.

13. Fokkens WJ, Lund VJ, Mullol J, Bachert C, Alobid I, Baroody F, Cohen N, Cervin A, Douglas R, Gevaert P, et al: EPOS 2012: European position paper on rhinosinusitis and nasal polyps 2012. A summary for otorhinolaryngologists. Rhinology 50: 1-12, 2012.

14. Zhang N, Van Zele T, Perez-Novo C, Van Bruaene N, Holtappels G, DeRuyck N, Van Cauwenberge P and Bachert C: Different types of T-effector cells orchestrate mucosal inflammation in chronic sinus disease. J Allergy Clin Immunol 122: 961-968, 2008.

15. Sheahan P, Ahn CN, Harvey RJ, Wise SK, Mulligan RM, Lathers DM and Schlosser RJ: Local IgE production in nonatopic nasal polyposis. J Otolaryngol Head Neck Surg 39: 45-51, 2010.

16. Bonfils P, Badoual C, Bonfils NA, Gallas D and Malinvaud D: Eosinophil infiltration of nasal polyps in patients with nasal polyposis Role in clinical evolution after medical and surgical treatment. J Laryngol Otol 123: 509-516, 2009.

17. Asero R and Bottazzi G: Nasal polyposis: A study of its association with airborne allergen hypersensitivity. Ann Allergy Asthma Immunol 86: 283-285, 2001.

18. Kirtsreesakul V and Atchariyasathian V: Nasal polyposis: Role of allergy on therapeutic response of eosinophil- and noneosinophil-dominated inflammation. Am J Rhinol 20: 95-100, 2006. 
19. Collins MM, Loughran S, Davidson P and Wilson JA: Nasal polyposis: Prevalence of positive food and inhalant skin tests. Otolaryngol Head Neck Surg 135: 680-683, 2006.

20. Kennedy JL and Borish L: Chronic sinusitis pathophysiology: The role of allergy. Am J Rhinol Allergy 27: 367-371, 2013.

21. Erbek SS, Erbek S, Topal O and Cakmak O: The role of allergy in the severity of nasal polyposis. Am J Rhinol 21: 686-690, 2007.

22. Nguyen LH, Fakhri S, Frenkiel S and Hamid QA: Molecular immunology and immunotherapy for chronic sinusitis. Curr Allergy Asthma Rep 3: 505-512, 2003.

23. Akdis CA, Bachert C, Cingi C, Dykewicz MS, Hellings PW, Naclerio RM, Schleimer RP and Ledford D: Endotypes and phenotypes of chronic rhinosinusitis: A PRACTALL document of the European Academy of Allergy and Clinical Immunology and the American Academy of Allergy, Asthma \& Immunology. JAllergy Clin Immunol 131: 1479-1490, 2013.

24. Ouyang Y, Fan E, Li Y, Wang X and Zhang L: Clinical characteristics and expression of thymic stromal lymphopoetin in eosinophilic and non-eosinophilic chronic rhinosinusitis. ORL J Otorhinolaryngol Relat Spec 75: 37-45, 2013.

25. Hu Y,Cao PP, Liang GT, Cui YH and LiuZ: Diagnostic significance of blood eosinophil count in eosinophilic chronic rhinosinusitis with nasal polyps in Chinese adults. Laryngoscope 122: 498-503, 2012.

26. Suzuki H and Ikeda K: Mode of action of long-term low-dose macrolide therapy for chronic sinusitis in the light of neutrophil recruitment. Curr Drug Targets Inflamm Allergy 1: 117-126, 2002.

27. Ikeda K, Shiozawa A, Ono N, Kusunoki T, Hirotsu M, Homma H, Saitoh T and Murata J: Subclassification of chronic rhinosinusitis with nasal polyp based on eosinophil and neutrophil. Laryngoscope 123: E1-E9, 2013.
28. Bachert C, Zhang N, Holtappels G, De Lobel L, van Cauwenberge P, Liu S, Lin P, Bousquet J and Van Steen K: Presence of IL-5 protein and IgE antibodies to staphylococcal enterotoxins in nasal polyps is associated with comorbid asthma. J Allergy Clin Immunol 126: 962-968, 968.e1-e6, 2010.

29. Sejima T, Holtappels G, Kikuchi H, Imayoshi S, Ichimura K and Bachert C: Cytokine profiles in Japanese patients with chronic rhinosinusitis. Allergol Int 61: 115-122, 2012

30. Cao PP, Li HB, Wang BF, Wang SB, You XJ, Cui YH, Wang DY, Desrosiers $\mathrm{M}$ and Liu Z: Distinct immunopathologic characteristics of various types of chronic rhinosinusitis in adult Chinese. J Allergy Clin Immunol 124: 478-484, 484.e1-e2, 2009.

31. Cao PP, Zhang YN, Liao B, Ma J, Wang BF, Wang H, Zeng M, Liu WH, Schleimer RP and Liu Z: Increased local IgE production induced by common aeroallergens and phenotypic alteration of mast cells in Chinese eosinophilic, but not non-eosinophilic, chronic rhinosinusitis with nasal polyps. Clin Exp Allergy 44: 690-700, 2014

32. Tripathi A, Conley DB, Grammes LC, Ditto AM, Lowery MM, Seiberling KA, Yarnold PA, Zeifer B and Kern RC: Immunoglobulin E to staphylococcal and streptococcal toxins in patients with chronic sinusitis/nasal polyposis. Laryngoscope 114: 1822-1826, 2004.

33. Zhang N, Holtappels G, Claeys C, Huang G, van Cauwenberge P and Bachert C: Pattern of inflammation and impact of Staphylococcus aureus enterotoxins in nasal polyps from southern China. Am J Rhinol 20: 445-450, 2006.

34. Tripathi A, Kern R, Conley DB, Seiberling K, Klemens JC, Harris KE, Suh L, Huang J and Grammer LC: Staphylococcal exotoxins and nasal polyposis: Analysis of systemic and local responses. Am J Rhinol 19: 327-333, 2005. 